\title{
RAPID DETERMINATION OF THE SICKLE CELL TRAIT BY THE USE OF A REDUCING AGENT
}

\author{
BY
}

\author{
A. W. WILLIAMS AND J. P. MACKEY
}

Colonial Medical Service, Dar-es-Salaam, Tanganyika

Various attempts have been made to find a more satisfactory method for the determination of the sickle cell trait than the sealed wet preparation, the deficiencies of which are well known. There is need for a rapid simple technique, giving a clearcut reading, which will serve for the rapid exclusion of sickle cell anaemia in clinical practice and also for the purpose of uniform survey work. The various techniques have been summarized by Diggs and Pettit (1940) and by Singer and Robin (1948). Because of the speed of the reaction the rapid " bacteriologic test" devised by the latter workers is an advance over previous methods; but its increased complexity is a disadvantage. For example, a living culture of Bacillus subtilis and an incubator are not available in the clinical side-room where a test of this nature is most wanted.

The original observation of Hahn (1928), confirmed and elaborated by Sherman (1940), established that the phenomenon of sickling only occurs in the blood of people with the sickle cell trait when the corpuscular haemoglobin is in a reduced or dissociated state, the change being accelerated by a low $p \mathrm{H}$. Search was therefore made for an acid reducing agent which, while causing no damage to the red cell in isotonic solution, would bring about rapid dissociation of oxyhaemoglobin and so reveal sickling in a short time.

The experience of one of us in preparing haemoglobin solutions for teaching purposes suggested sodium hydrosulphite (dithionite, $\mathrm{Na}_{2} \mathrm{~S}_{2} \mathrm{O}_{4}$ ) as a likely substance, and this was selected for trial. A 2.1 per cent aqueous solution was found to be isotonic and to have a $p H$ of 6.4. It has proved an excellent and reliable reagent for the quick demonstration of the sickling trait.

\section{Method}

Sodium hydrosulphite is a powerful reducing agent which decomposes in moist air to a mixture of sodium thiosulphate and sodium sulphite. A solution, therefore, will not keep indefinitely. For the preparation of the solution distilled water which has been freshly boiled and allowed to cool is used. The 2.1 per cent solution is made once a week, and kept in small tubes with a covering seal of liquid paraffin. Once the paraffin seal is removed, the tube should be discarded at the end of the day.

Technique and Reading of Test.-A drop of the isotonic sodium hydrosulphite solution is taken up in a finely drawn Pasteur pipette, and about one-sixth of this quantity of blood (from either a skin prick or venepuncture) is drawn up in apposition to the column of fluid. The whole is then expelled on to a clean slide and rapidly mixed. The slide is inverted on to a coverslip and examined under the $1 / 6$ objective. It is not necessary to seal the coverslip with vaseline. The same pipette may be rinsed in normal saline and used for any number of patients in rapid succession.

If the sickle cell trait is present, sickling will occur in from 3 to 15 minutes, and rapidly become definite and generalized. It tends to appear earliest in cells near the edge of the coverslip, possibly due to the effect of surface tension on the rate of reaction. If there is no sickling after 20 minutes the blood can be pronounced negative for the sickle cell trait. Sickling never occurs in normal blood, though the red cells may undergo crenation and haemolyse after half an hour.

Comparison with Sealed Wet Preparation.-One hundred African schoolboys were examined by both the sealed wet preparation, read at 48 hours, and the sodium hydrosulphite technique. There was complete agreement, both as to positives and negatives, between the two methods. (The numerical findings form part of a survey which will be published separately.) The sodium hydrosulphite technique always gave clear-cut results within 20 minutes, whereas with the sealed wet preparation the reading was sometimes still doubtful after 24 hours.

\section{Summary}

A technique is described for routine determination of the sickling trait, employing a reducing agent to produce rapid dissociation of oxyhaemoglobin. A drop of a freshly prepared 2.1 per cent aqueous solution of sodium hydrosulphite is mixed 
with about one-sixth of its volume of blood. The suspension of red cells is observed under a coverslip for up to 20 minutes. No seal is necessary. In speed, simplicity, and reliability this procedure is considered superior to other methods in use at the present time.

Our thanks are due to Dr. W. D. Raymond, Government chemist, Tanganyika, for his assistance and advice in this investigation, and to the Director of Medical Services, Tanganyika, for permission to publish.

\section{REFERENCES}

Diggs, L. W., and Pettit, V. D. (1940). J. Lab. clin. Med., 25, 1106은 Hahn, E. V. (1928). Amer. J. med. Scl., 176, 206.

Sherman, I. J. (1940). Bull. Johns Hopk. Hosp., 67, 309.

Singer, K., and Robin, S. (1948). J. Amer. med. Ass., 136, 1021.

Shortly after submitting this paper for publication, the work op Thomas and Stetson (Johns Hopk. Hosp. Bull., 1948, 83, 176) came to our notice. Of sulphydryl compounds which they tried, a saturated solution of hydrogen sulphide was found to be the most reliable ang rapidly acting agent for the demonstration of sickling. While theres is probably little to choose between the two reagents in their efficiency as indicators of sickling, sodium hydrosulphite has practical advan tages as to odour, stability, and preparation of the solution. 